\title{
Documentación y producción artística en la cultura Yucateca. Archivo de experiencias.
}

\section{Documentation and artistic production in the Yucatecan culture. Archive of experiences.}

\author{
TIPO DE TRABAJO: \\ Comunicación. \\ PALABRAS CLAVE: \\ Producción artística, local, experimentación, registro, memoria.
}

KEY WORDS:

Artistic production, Local, Experimentation, Record, Memory.

RESUMEN.

Esta comunicación presenta y analiza un proyecto artístico llevado a cabo por ambas autoras durante una estancia en Yucatán, México en 2016. El proyecto, titulado: Retrato en 25. Cartografiando memorias entre Tunkás e Izamal se mueve por la necesidad de conocer y explorar diferentes lugares a través de la experiencia personal, descubrir las raíces de lo micropolítico y analizar la implicación del artista como agente externo en un contexto local 'desconocido'. A través de este trabajo encontramos la posibilidad de participar en un contexto diferente, interpretando los códigos que construyen la memoria, la identidad cultural y los espacios comunes. Se puso en marcha mediante estrategias de las ramas antropológica y etnográfica que, desde una perspectiva diletante y liviana, ofrecen el terreno de juegos adecuado para realizar entrevistas, trabajo de campo y recolección de objetos y documental. Estrategias que a la vez nos permiten desarrollar un trabajo plástico en el que la fotografía, el audiovisual, la acción y la edición son parte esencial para la interpretación y reinterpretación de los individuos y el contexto en el que habitamos. Recorremos el espacio físico y recolectamos objetos que rozan lo insignificante o desapercibido para ponerlos bajo otra óptica. En el transcurso de estas acciones se despierta el reconocimiento de cuestiones micropolíticas como síntoma de posiciones más globales y a la vez el descubrir cómo afectan las realidades globales a un contexto local.

\section{ABSTRACT.}

This paper presents and analyzes an artistic project conducted by both authors during a residence in Yucatán, Mexico, 2016. The project, entitled Portrait in 25. Mapping Memories between Tunkás and Izamal is driven by the need to know and explore different places through personal experiences, to discover micropolitical sources and analyze the artist's involvement as an external agent in an 'unknown' local context. Through this work we find a chance to participate in a different context and convey the meaning of the set of rules that frame their memory, cultural identity and common spaces. It was launched through anthropological and ethnographic strategies, in a rather light and amateur sense, to offer a suitable playground for interviews, fieldwork and, the gathering of objects and documents. All at once these strategies allow us to develop a plastic work in which photography, audiovisual, performance and editing are an essential part for the interpretation and reinterpretation of the individuals, and the context we inhabit. We go across physical space and collect objects seemingly insignificant or unnoticed to put them under another point of view. In the course of these actions the recognition of micropolitical issues is awakened as a symptom of global positions and, at the same time, we discover how global realities affect the local context. 


\section{CONTENIDO.}

\section{Introducción.}

El proyecto Retrato en 25. Cartografiando memorias entre Tunkás e Izamal surgió de la necesidad de conocer y experimentar otros lugares de primera mano y de la manera más amplia, es decir, abriéndonos a la red de conexiones entre hechos, personas y cosas que nos pudiera ofrecer el lugar, a su multiplicidad. Recorrer y conocer el lugar tratando de desentrañar ciertas claves, ¿De qué manera describen, interpretan y representan estas comunidades su historia o memoria colectiva? ¿Qué códigos sociales, problemáticas locales y micropolíticas atraviesan en cuerpo de las personas en estos lugares? ¿Qué modos de acción se producen por parte de la población para mediar con diversas polaridades políticas? ¿Qué visiones pueden aportar las narrativas artísticas a los planteamientos locales?

\section{Pre. Antes del viaje.}

La motivación del proyecto surgió tras advertir del conflicto que generó el desplazamiento de los trabajadores de las zonas rurales y la crisis económica generalizada a partir del cese de las haciendas henequeneras ${ }^{1}$. Entendimos el desarrollo de la industria henequenera y su desplome, como una probable fuente de tensión micropolítica. Investigar y analizar la documentación, recoger y catalogar objetos y registrar testimonios sonoros al respecto serían los objetivos a alcanzar.

Esta quizá ambiciosa empresa se llevaría a cabo en 25 días de estancia y con esta intención en mente decidimos comenzar la investigación en España. La razón de esto era, por un lado, comparar el conocimiento adquirido a priori con la experiencia a vivir en Yucatán y por otro, definir el espacio geográfico en el que trabajaríamos. Se trataría, por tanto, una vez ahí, de desarrollar nuestras ideas con un trabajo de campo cuya base de operaciones se situaría en una hacienda en la zona limítrofe entre las municipalidades de Izamal y Tunkás.

La manera de formalizar esto iría unida a dos experiencias. La primera un diario de campo / libro de artista que se planteaba como un archivo abierto, en el que plasmar todas las cuestiones tratadas. En él se daría salida a todas las investigaciones previas a nuestra llegada y cuestiones como la geografía del lugar, la historia de las haciendas, su relación con la industria del henequén, la economía local etc. Cuestiones que se incluirán dentro de una estructura taxonómica abierta. Estructura que a su vez permitiría analizar y generar relaciones entre los diferentes agentes activos de la zona, además de formar el cuerpo de un proceso de trabajo visual y conceptual. El archivo incluiría material de registro, fotografías de las zonas visitadas; textos en apoyo a la interpretación y desarrollo del discurso; dibujos, esquemas, objetos encontrados y en general, documentación sobre las intervenciones y recorridos.

La segunda experiencia albergaría los testimonios de las personas que habitan las haciendas de la zona. Buscábamos una visión familiar, no invasiva hacia la problemática local que plantea el cultivo del henequén. Qué significó para el contexto tanto social, como cultural y sus vínculos con el presente. Estos testimonios serían registrados en formato video para su posterior proyección y visionado.

\section{En Kankabal, las haciendas.}

Las haciendas fueron hasta mediados del siglo XX, los centros neurálgicos de la producción industrial en Yucatán. Con la aparición de la fibra sintética, la Industria Henequenera empezó a decaer y en consecuencia también la mayoría de las haciendas, o al menos aquellas que no optaron por una diversificación de la producción. A pesar de que existen varias iniciativas de remodelación, como las que se llevan a cabo en las que visitamos, muchas de ellas están abandonadas y sujetas a las inclemencias del tiempo.

Kankabal es el nombre de una antigua hacienda ganadera situada en la provincia de Yucatán, entre la municipalidad de Izamal, conocida por un rico patrimonio cultural y pasado histórico precolombino, y Tunkás, un pequeño pueblo obrero de unas pocas casas. La hacienda es ahora una base operativa de nuestros anfitriones, el grupo Noox ${ }^{2}$, que, entre otras cosas, promueven la creación artística mediante su trabajo de gestión en la residencia y en centros de exposiciones repartidos por el país. La estancia transcurre a lo largo de un mes, y una vez en el lugar la propuesta cambia.

Uno de los cambios fue la decisión de centrarnos en tres haciendas, San Diego Azcorra, Ticopó y Kankabal. Situadas entre la ciudad de Mérida y Tunkás, en un rango de 100 km de distancia. San Diego Azcorra se encuentra dentro del casco urbano de Mérida donde a principios del siglo XIX se dedicaba al cultivo y procesado del henequén. En su casa principal actualmente se ubica la galería Noox (Mérida) mientras que el resto de sus tierras han sido urbanizadas. La hacienda Ticopó, ubicada en la población con el mismo nombre también era henequenera. Esta se encuentra en proceso de restauración y todavía conservaba algunos espacios inalterados.

\footnotetext{
${ }^{1}$ El henequén o sisal, fue durante finales del siglo XIX y principios del XX la base económica de la provincia de Yucatán. Es una especie de agave que se utilizaba entre otras cosas para la producción de fibra. Una visión crítica al respecto de lo que supuso a nivel social la Industria Henequenera en Yucatán léase los artículos de John K. Turner, 2014.

${ }^{2} \mathrm{http}: / /$ www.noox.mx/residencia-de-arte-noox-kankabal
} 


\section{La experiencia.}

Para hacer un primer reconocimiento de los lugares dedicamos un tiempo a visitar hemerotecas y bibliotecas de la capital, donde encontramos información sobre el pasado de las haciendas. Después establecimos unos ejercicios creativos condicionados que nos permitieran registrar los espacios y conocer a las personas que los habitaban, bajo una misma estructura experimental y documental. Una de las ideas que subyace a esto es también la de encontrar material con el que poder trabajar.

$$
\text { a. Reglas del juego. }
$$

La forma en que decidimos enfrentarnos a los espacios se puede asociar con la deriva propuesta por las vanguardias si la entendemos como: "La materialización de un modo alternativo de habitar la ciudad..." o el espacio (Careri, 2009). Mediante ejercicios lúdicocreativos propondríamos ver el espacio desde una perspectiva diferente, que no solo lo topografiara, pero también forzara la presencia en el lugar mediante la sistematización de una acción, generando una referencia mnemónica concreta. A su vez, esto dio lugar a que viéramos los lugares desde todos los ángulos, que des-subjetiváramos nuestra visión sobre un edificio o una arquitectura que propone sus propios lugares de tránsito. Nos enseñó la imposibilidad de una perspectiva total, tanto en la imagen como en la experiencia del lugar.

Respondieron a esta reflexión también los ejercicios de recogida de objetos (restringida a tres), la idea de cartografiar y situar los espacios, realizando una serie de fotografías panorámicas desde los cuatro puntos cardinales, la recogida de muestras de tierra de los puntos cardinales, (doce en total) y la acción de nuestros cuerpos en el espacio arquitectónico, (recogida en formato videográfico).

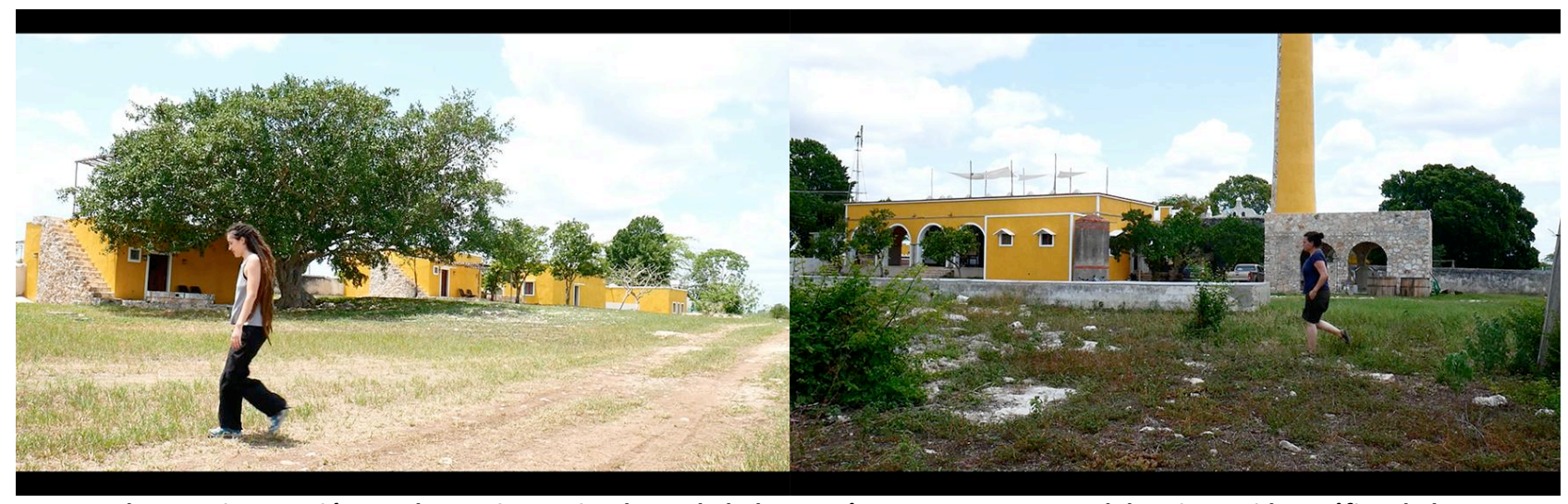

Proceso de experimentación en el espacio. Hacienda Kankabal, Yucatán, 2016. Fotograma del registro videográfico de las autoras.

En el proceso de documentación extrajimos moldes de algunos objetos especialmente destacados de las haciendas, la mano de una figura religiosa perteneciente a una capilla en el interior de la hacienda Kankabal, letras de una inscripción tallada en mármol en la hacienda Azcorra o una etiqueta identificativa en relieve de una de las antiguas bombas de agua de la fábrica de fibras henequenera de Ticopó. El resultado de este proceso se materializó en resina y se sumó al archivo. También se añadieron los objetos encontrados, como una pieza que correspondía a la balaustrada de la antigua entrada de Ticopó, una antigua teja de origen francés usada para el techo, una oxidada hamaquera de Kankabal, losetas con diferentes motivos y el cañón de un arma desechado y oxidado. El resultado de la acumulación de estos objetos se asemejaba a una de las vitrinas del Museo de Historia Natural.

Esta primera experiencia de trabajo de campo se plasmó finalmente sobre cuatro libros de artista. En cada uno de ellos se concretó un ejercicio de documentación diferente. El Libro I se componía de tres desplegables destinados a cada una de las haciendas. Entre collage, ilustraciones y fotografías, que resaltaban sus diferentes colores por medio de composiciones en ángulos rectos. El Libro I/ era un fotomontaje distribuido de manera circular sobre el papel, que recogía una visión de 360 desde los diferentes puntos cardinales de cada hacienda. El Libro III se contempló como un cuaderno de campo que registraba una serie de datos importantes sobre las haciendas, hechos históricos que se narraron en base a fotografías, ilustraciones, impresiones y recortes de periódico. El Libro IV se construyó como un único desplegable que mostraba en orden cronológico las cartografías utilizadas para reconocer el lugar, desde los mapas realizados en España a las relaciones geográficas desarrolladas después.

\section{b. Entrevistas.}

Utilizamos las visitas y los objetos encontrados como una herramienta para entablar un diálogo con los trabajadores de las haciendas. De una manera orgánica unas personas nos llevaron a otras y acabamos por realizar una serie de entrevistas con personas de origen 
maya cuya tradición familiar estaba relacionada con las haciendas en más de dos generaciones. Las preguntas giraban en torno al trabajo que realizaban, a sus costumbres y tradiciones y a su conocimiento histórico del lugar. El desarrollo de las entrevistas pretendía seguir una lógica emic, (Harris, 1976) en la que priorizamos cómo explicaban las personas nativas el significado de las costumbres o de los hechos.

Gracias a su participación fuimos capaces de distinguir algunas costumbres y convenciones sociales. Nos ayudaron a contextualizar temporal y espacialmente algunos de los objetos encontrados. Nos describieron tradiciones y mitos arraigados en su cultura. Al igual que cantaron algunas canciones populares y nos enseñaron palabras y expresiones mayas.

\section{c. Mesa/vitrina.}

Debido a las renovaciones que se estaban realizando en las haciendas, muchas piezas pertenecientes al antiguo decorado y carpintería (puertas, ventanas...) se estaban desechando. Estas piezas llamaron nuestro atención y con ellas proyectamos la construcción de una mesa-vitrina en la que desplegar los objetos y trabajos realizados durante la estancia. Tomaba la forma de una de las puertas principales de la propia hacienda.

Dos grandes puertas que conservaban sus contraventanas nos sirvieron para alojar los libros, las muestras de tierra y demás objetos etiquetados. Colocados en un espacio interior que, gracias a las bisagras, se podía abrir y cerrar, llamaba a la interacción. En uno de los bordes, colocamos unos auriculares que reproducían un montaje sonoro con los diferentes testimonios registrados.

La mesa se expuso en la sala central de la casa principal de la hacienda. En cierta medida evocaba una relación de poder. La mesa entendida como alegoría de lo próspero, los grandes hacendados, chocaba con su interpretación como muestrario o repositorio de restos históricos o testimonios archivados. Esta relación nos resultó interesante por el hecho de que la monumentalidad del objeto legitimaba la interpretación de los vestigios como historia viva del lugar, poniendo de manifiesto transversalmente cierta artificiosidad en la construcción de un discurso histórico, no exento, al fin y al cabo, de relaciones de poder.

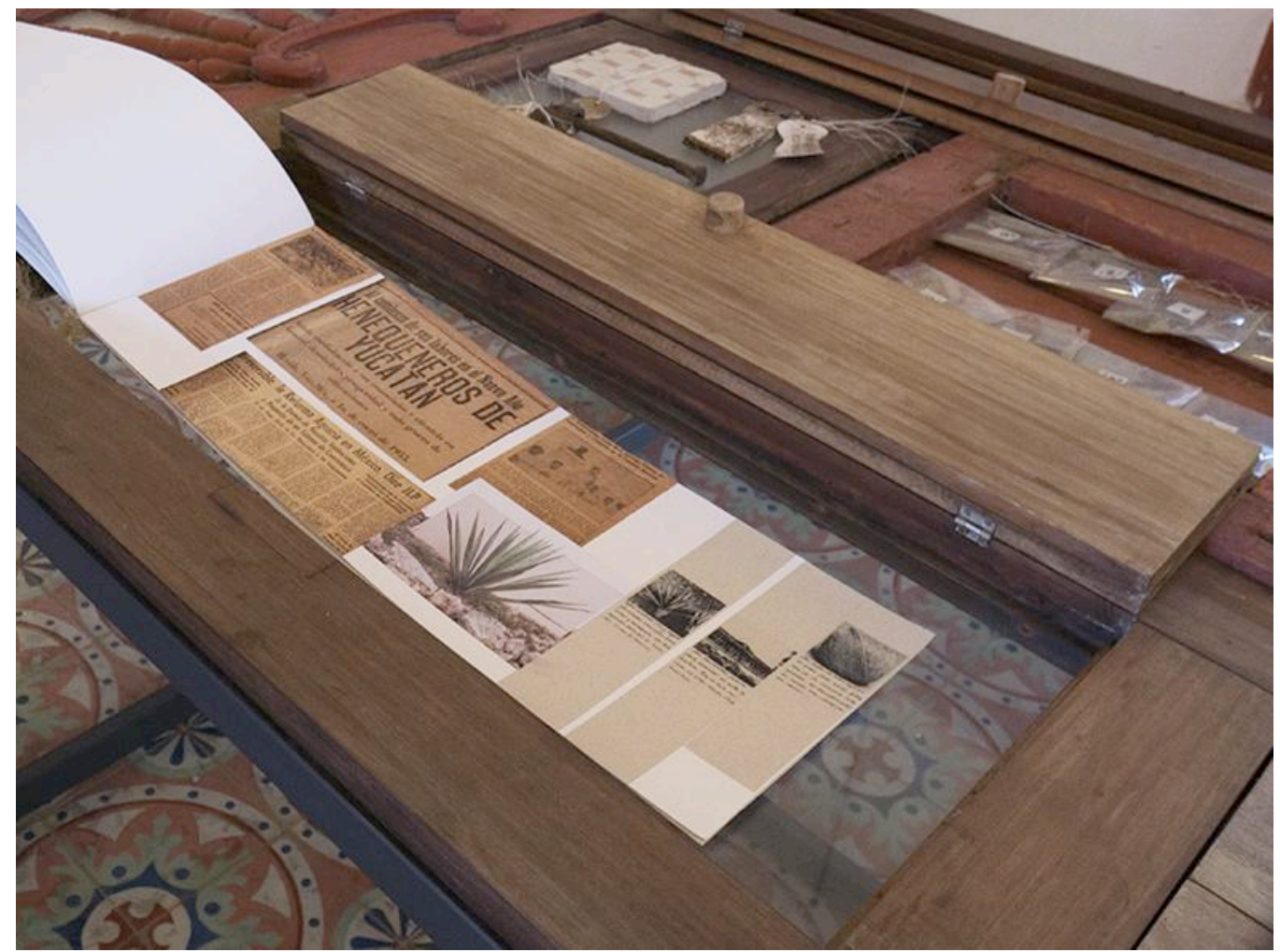

Detalle del Libro III colocado sobre una de las puertas de la mesa/vitrina. Hacienda Kankabal, Yucatán, 2016. Fotografía de las autoras. 


\section{d. Video-Mapping.}

En la segunda experiencia, o fase, de nuestro proyecto se desarrolló el proceso de documentación sonora y videográfica. Se realizaron un mínimo de cinco registros sonoros de una duración de veinticinco segundos, ubicados en diversas partes de las haciendas. En cuanto al registro videográfico se siguió el mismo patrón, un mínimo de dos grabaciones en video de la misma duración. Asimismo, se grabaron diversos planos secuencia tomados desde cada uno de los puntos cardinales, tomas referentes a la identidad cultural, sus vestimentas, fiestas, comidas y labores artesanales, como por ejemplo la elaboración del mezcal o el uso de un traje tradicional de gala, el hipil, y su simbología. Para su edición utilizamos el montaje, entendido como una técnica narrativa y de interpretación de los códigos. Su uso establece una serie de relaciones asociativas interesantes que interpretan acciones y enunciados bajo una misma óptica.

Se nos brindó la oportunidad de adaptar el montaje en un video-mapping proyectado sobre la fachada de la hacienda Kankabal. De esta técnica se desprende cierta espectacularidad y al proyectarse sobre el mismo objeto de nuestra investigación se estableció una relación metonímica. La arquitectura en sí como representación de las manifestaciones que de ella se desprendían desde el principio.

\section{Post. Reflexiones a posteriori.}

Nos hemos encontrado con una sociedad muy heterogénea, en la que tanto las diferencias sociales, como las costumbres de cada parte de la población o el uso de diferentes lenguas nativas nos da diferentes aproximaciones a muy distintas herencias culturales. Una propuesta que busque descubrir los códigos sociales, las problemáticas y micropolíticas no solo necesita de más tiempo y asimilación, también debe dar fe de la multiplicidad y transculturalidad en la que está inmersa.

Derivado de los ejercicios de registro y las entrevistas nos dimos cuenta de que habíamos construido alrededor de la búsqueda de información, una serie de presuposiciones. Una de ellas fue asumir que había una conciencia individual arraigada de la memoria histórica o colectiva y que sería de fácil acceso. Las experiencias personales y de antepasados sobre el trabajo en las haciendas no fue una cuestión sencilla de desvelar, pues están basadas principalmente en una tradición oral y en el recuerdo, no siempre grato de contar. No se nos escapa que nuestra presencia cambiaba las condiciones habituales de los entrevistados, como tampoco que la suya influye en nuestras presunciones.

Vimos en nuestra actuación ciertas limitaciones causadas por, entre otras cosas, la incapacidad de casar una abstracción de la memoria con los testimonios concretos, el corto tiempo de nuestra estancia, que impedirían una profundización mayor en estos temas, o la existencia de barreras idiomáticas, la lengua materna de estas personas es el maya. Aun así, los registros nos dieron pie a hacer un montaje audiovisual que rescataba ciertos datos de los testimonios encontrados estableciendo asociaciones entre acontecimientos que en principio no se habrían desvelado. En este sentido vemos que el conocimiento generado a partir de asociaciones visuales y sonoras (en una descontextualización y estetización del documento), puede ayudar a generar cierta conexión entre diferentes realidades, que traspasan las barreras de lo local y lo global.

Lo cierto es que los ejercicios de registro permanecen, no solo como una experiencia azarosa, sino como experimento que siguiendo un orden determinado, establece una construcción nítida en la memoria. Aun así y visto desde cierta distancia somos partidarias de la tesis de Sven Spieker que dice que "Los archivos no registran tanto la experiencia como su ausencia; señalan el punto en el que falta una experiencia del lugar que le es propio, y lo que se nos regresa en un archivo puede muy bien ser algo que en primer lugar nunca fue nuestro" ${ }^{3}$ (Spieker 2008:3) La experiencia en Yucatán y su materialización en un orden archivístico nos desvela así cuestiones que no nos habíamos planteado y que seguimos resolviendo.

\section{FUENTES REFERENCIALES.}

CARERI, F. 2009. Walkscapes. El andar como práctica estética. Barcelona: Gustavo Gili. ISBN 9878425218415.

HARRIS, M. 1976. History and Significance of the Emic/Etic Distinction. Annual Review of Anthropology, volumen (5) $329-350$. Disponible en : http://www.jstor.org/stable/2949316

SPIEKER, S. 2008. The Big Archive. Art from Bureaucracy. Londres: The Mit Press. ISBN 9780262195706.

TURNER, K. 2014. México Bárbaro. México: Ed. Méxicanos Unidos S.A. ISBN 9786071411525.

\footnotetext{
${ }^{3}$ Traducido por las autoras, la cita original es: "Archives do not record experience so much as its absence; they mark the point where an experience is missing from its proper place, and what is returned to us in an archive may well be something we never possessed in the first place." En Spieker, 2008:3
} 